\title{
Peer Review 2.0: Herausforderungen und Chancen der wissenschaftlichen Qualitätskontrolle im Zeitalter der Cyber-Wissenschaft
}

\section{EINLEITUNG}

$\mathrm{D}$ e neuen Informations- und Kommunikationstechnologien haben zweifellos einen massiven Einfluss auf Wissenschaft und Forschung. Akademikerinnen und Akademiker tauschen E-Mails aus, partizipieren in Online-Debatten, kooperieren und nutzen Datenbanken auf Distanz, simulieren und modellieren Realitäten auf ihren Computern und lehren über das Web. Eine der augenscheinlichsten Auswirkungen der Entwicklung hin zur "Cyber-Wissenschaft" ist im Bereich des wissenschaftlichen Publikationssystems erkennbar. Akademisches Publizieren ist nicht mehr das, was es vor dem Aufkommen des Internets war:

Zahlreiche traditionelle Zeitschriften sind nun auch als Online-Ausgabe erhältlich, in vielen Feldern entstanden innovative elektronische Journale, riesige digitale Arbeitspapierarchive gewähren in einem frühen Stadium Zugang zu Forschungsliteratur und wissenschaftliche Bibliotheken werden langsam zu „Cybertheken“, die Zugang zu digitalen Quellen aller Art bieten. Zudem können wir neue Formen wissenschaftlichen Publizierens ausmachen, welche in der traditionellen papierbasierten Welt nicht möglich gewesen wären, da sie nur in digitalen Formaten realisierbar sind. Hypertexte präsentieren Wissen auf neue Weise, Multimedia verbessert die Wege der Informationsvermittlung an die Leser und der Austausch von Forschungsergebnissen über Datenbanken wird immer üblicher. Kurz gesagt: Die herkömmlichen wissenschaftlichen

I Michael Nentwich: Cyberscience: Research in the Age of the Internet, Vienna, 2003, <http://hw.oeaw.ac.at/3188-7>. 
Kommunikationsformen erfahren durch die neuen technologischen Möglichkeiten einen fundamentalen Wandel. Eine der meistdiskutierten Herausforderungen ist in diesem Zusammenhang die Qualität. Während die etablierten Verfahren der wissenschaftlichen Qualitätskontrolle das Kernstück des heutigen Forschungssystems darstellen, werden diese Traditionen durch das elektronische Publizieren in den Augen vieler infrage gestellt:

Zum einen ebnen die neuen Technologien den Weg zu neuen Begutachtungsformen, insbesondere zu Systemen mit der Funktion des open peer review (d. h. einer offen zugänglichen Bewertung und Kommentierung durch die Fachkollegen). Dies wird in Blogs bisweilen in Anlehnung an den Begriff „Web 2.0" unter „Peer Review 2.0" verhandelt, ist aber in der Fachwelt noch nicht etabliert. ${ }^{2}$ Zum anderen hat die Möglichkeit zum Selbstpublizieren zu einem massiven Aufkommen online verfügbarer Papiere geführt, oft ohne große - oder gar ohne jegliche - Qualitätskontrolle.

Im vorliegenden Kapitel werden diese Entwicklungen der Cyber-Wissenschaft vorgestellt und eine Einschätzung bezüglich der Zukunft der Qualitätskontrolle als dem wichtigsten Fundament der Forschung gegeben. Dabei soll insbesondere das weitverbreitete Argument, ein Mehr an Online-Publikationen führe zwangsläufig zu einer Verschlechterung der Qualität, diskutiert werden. Zudem sollen mögliche Probleme bei der Umsetzung angesprochen werden.

ARTEN DER CYBER-QUALITÄTSKONTROLLE

Zusätzlich zu den vielen traditionellen Varianten akademischer Qualitätskontrolle (insbesondere peer review) entstehen im Zeitalter der Cyber-Wissenschaft zahlreiche neue Möglichkeiten, die vielleicht zu einem neuen System der Qualitätskontrolle führen werden.

Neue Formen der Ex-ante-Qualitätskontrolle

Das kommunikative Potenzial des Internets, insbesondere das von WWW-

2 Siehe z. B. <users.dimi.uniud.it/ -stefano.mizzaro/research/PeerReview2.o. html>, $<$ gunther-eysenbach.blogspot.com/2008/03/medicine-20-congress-websitelaunched.html >, <ncsi.iisc.ernet.in/pipermail/lis-forum/2006-June/004215.html>. 
Publikationen, kombiniert mit E-Mail und Webmailing ermöglicht Systeme mit offen zugänglicher Bewertung und Kommentierung durch die Fachkollegen. Im englischen Sprachraum sind diese weitläufig unter open peer review (bisweilen auch open peer commentary) bekannt. Diese neuen Begutachtungsformen kommen entweder in Reinform (I) oder gemischt mit traditionellen Bewertungssystemen (2) vor. Zusätzlich erlaubt das elektronische Publizieren differenzierte Bewertungsstandards (3).

(I) Open peer review: Während die Forschungsgemeinde im Allgemeinen traditionell erst nach dem Druck von einer Einreichung erfährt, wird diese bei Open-peer-review-Verfahren in elektronischen Zeitschriften bereits zuvor angekündigt; der Artikel wird auf den Server der Zeitschrift hochgeladen und ist für alle einsehbar.

Bei Überarbeitungen (vom ursprünglich eingereichten bis hin zum letztlich publizierten Text) sind auch die Zwischenschritte erkennbar. Zudem wählen weder die Herausgeber noch die Gutachter aus, sondern eröffnen eine offene Debatte zum Manuskript. Selbsternannte Gutachter ${ }^{3}$ kommentieren online die Güte des Papiers. ${ }^{4}$

In der Regel werden weder die Identität der Autorinnen und Autoren noch die der öffentlichen Gutachter verborgen. Zwar gibt es durchaus gute Gründe für Anonymität, jedoch erscheint es in diesem Kontext unpassend, wichtige Beurteilungen über die Arbeit anderer im Geheimen zu äußern. ${ }^{5}$ Obwohl Anonymität zwar technisch machbar wäre, wird sie bis auf wenige

3 Vgl. Tamara Sumner / Simon Buckingham Shum: From Documents to Discourse: Shifting Conceptions of Scholarly Publishing, in: CHI '98, I8.-23.4.1998, Los Angeles 1997, <http://citeseerx.ist.psu.edu/viewdoc/download?doi=IO.I.I.27 $.6407 \&$ rep=repi\&type $=$ pdf $>$.

4 Ein Beispiel dafür ist <www.philica.com>. Hier kann prinzipiell jede/r Reviews schreiben. Rezensenten können jedoch auch einen Beleg über ihren akademischen Status einreichen, wodurch ihre Bewertungen ein höheres Gewicht erhalten. Demgegenüber werden „unconfirmed members“, also solche ohne „confirmed academic status“, zwar prinzipiell akzeptiert, aber ihnen wird kaum Beachtung geschenkt.

5 Vgl. Richard Smith: Editorial: Opening up BMJ peer review. A beginning that should lead to complete transparency, in: British Medical Journal online, 318 (1999), 4-5, <http://bmj.com/cgi/content/full/318/7175/4>. 
Ausnahmen ${ }^{6}$ kaum realisiert, da sie dem ansonsten offenen Charakter des Verfahrens zuwiderliefe.

Die Philosophie hinter open peer review ist im Grunde die Erwartung, das Endergebnis würde mit steigender Teilnehmerzahl besser. Ein offenes, nichtanonymes Verfahren sei „demokratischer" und weniger von einzelnen Verlegern oder Gremien bestimmt. Darüber hinaus stünden die Autoren am Ende nicht lediglich vor einer bereits gefällten Entscheidung, sondern sind in der Lage, am Bewertungsprozess interaktiv teilzunehmen. Während es sich beim traditionellen Begutachtungsmodell eher um eine individuelle Aktivität handelt, könnte das offene Verfahren zu einem Diskurs führen, der Ideen formt und verfeinert.

(2) Mischsysteme: Mittels Kombination von traditioneller Begutachtung und open peer review erproben Verleger innovativer Zeitschriften eine Reihe neuer Modelle. Man überträgt beispielsweise die Idee der Offenheit und Transparenz auf den Begutachtungsprozess. So lassen sich etwa die Kommentare der Gutachter veröffentlichen und interaktiv nutzen. ${ }^{7}$ In einer anderen Alternative könnte eine vorab anonyme Begutachtung mit einer Open-peer-review-Phase zu einem späteren Zeitpunkt kombiniert werden. ${ }^{8}$

(3) Differenzierte Bewertungsstandards: Die Umgebung digitaler Publikationen ermöglicht Qualitätsstandards auf verschiedenen Ebenen. Zwei Vorschläge sollen an dieser Stelle die differenzierten Bewertungsstandards illustrieren.

(a) Im Kontext eines Szenarios, in dem wissenschaftliche Kommunikation über Hypertexte erfolgt, können Bewertungsstandards je nach Hypertextmodul9 unterschiedlich gesetzt werden. So könnten etwa bei sog. „fixen“ Hyper-

6 Z.B. Atmospheric Chemistry and Physics (APC), <www.atmospheric-chemistryand-physics.net>.

7 Dies wird z. B. bei <www.biology-direct.com> so gemacht. Siehe: Elisabeth Davenport / Blaise Cronin: Hypertext and the Conduct of Science, in: Journal of Documentation, 46 (1990), 3, S. 175-192.

8 Z.B. Journal of Interactive Media in Education (JIME), <ww-jime.open.ac.uk>.

9 Vgl. Joost Kircz / Hans Roosendaal: Understanding and shaping scientific information transfer: Electronic publishing in science. Proceedings of the joint ICSU Press - UNESCO Expert Conference, Paris 1996, S. I06-116, <http://www.science.uva.nl/projects/commphys/papers/unescom.htm>. 
text-Modulen, also solchen, die einzelne typische Teile eines wissenschaftlichen Texts enthalten, diese differenziert bewertet werden. ${ }^{\text {IO }}$

(b) Elektronische Archive könnten weiters bestimmten wissenschaftlichen Inhalten eine neue Chance geben. Denn manche Beiträge wurden bisher in traditionellen Zeitschriften abgelehnt - nicht aufgrund ihrer mangelhaften Qualität, sondern weil sie nicht „im Einklang mit der Linie“ der Zeitschrift standen oder schlichtweg zu lang waren. Für die Zeitschriftenfamilie von BioMedCentral wurde nach einem Vorschlag von Varmus bereits 1999 eine spezielle Zeitschrift gegründet, die auch Beschreibungen von Experimenten mit nicht eindeutigen oder "negativen " Ergebnissen veröffentlicht. ${ }^{\text {II }}$ Zwar wird derartiges Material in den meisten aktuellen Zeitschriften in der Regel nicht publiziert, dennoch ist es für Akademiker mit ähnlichen Forschungsansätzen durchaus nützlich. In beiden Fällen würde die Qualität kontrolliert, jedoch nicht auf gleicher Ebene. So wird der Zugang zu innovativem oder anderweitig nützlichem Material ermöglicht.

\section{Neue Formen der Ex-post-Qualitätskontrolle}

In der traditionellen papierbasierten Publikationsumgebung ist die Qualitätskontrolle nur ex ante durchführbar. Selbst wenn etwas fehlerhaft oder verbesserungsbedürftig ist, bleibt es ein Teil des veröffentlichten Wissensstandes: Man kann einen gedruckten und weltweit bereitgestellten Text weder ex post löschen, noch kann man ihn als „mit Vorsicht zu behandeln“ deklarieren. Cyber-Wissenschaft bietet hingegen eine Reihe von Ex-post-Mechanismen, die genau dazu in der Lage sind. Bereits heute (umso mehr noch in Zukunft) werden Publikationen überwiegend mithilfe onlinegestellter bibliographischer Datenbanken gesucht und nicht mehr, indem Bücherregale durchstöbert werden. Ein einfacher, aber gefährlicher Weg zur Vermeidung falscher

Io Siehe z.B. die Methodenbeschreibung oder die Analyse des Ausgangswissensstandes, dazu Nentwich: Cyberscience, S. 264.

II Journal of Negative Results in Biomedicine, <www.jnrbm.com>. Siehe: Harold Varmus und andere: PubMed Central: An NIH-Operated Site for Electronic Distribution of Life Sciences Research Reports, 1998 (letzte Aktualisierung: 30.08.1999), <http://www.nih.gov/about/director/pubmedcentral/pmcprint. htm>. 
Resultate - wie auch immer diese zustande gekommen sein mögen - wäre daher die Entfernung eines Eintrags von der „ersten Schicht" der Datenbank. Dadurch wäre dieser nicht mehr sofort auffindbar.

In der digitalen Welt ergibt sich indessen eine weitere Alternative: Man könnte sowohl die Publikation als auch den bibliografischen Eintrag unberührt lassen, den Lesern (und auch den Autoren) jedoch die Möglichkeit zum Verfassen von Kommentaren unterschiedlicher Art bieten. Die nachfolgenden Lesenden könnten den Inhalt auf diese Weise für sich selbst einschätzen. ${ }^{\mathrm{I}}$

Im Folgenden sollen drei solche Mechanismen vorgestellt werden: Annotierung (I), Bewertung (2) und Analyse von Nutzungsverhalten (3).

(I) Online (ex post) Kommentierung bzw. Annotierung: Auch nach der Publikation kann durch Kommentare (oder Annotationen) der Leser noch Qualitätskontrolle stattfinden. Die Annotationen können einfach an den jeweiligen Artikel ,angefügt" werden. Eine derartige Qualitätskontrolle wäre sowohl für Selbstveröffentlichungen als auch für bereits in elektronischen Zeitschriften publizierte Artikel (unabhängig von der ursprünglichen Qualitätskontrolle) realisierbar. Die dazu nötige Technologie wurde schon entwickelt und wird derzeit erforscht. Die Kommentare werden zusammen mit dem Artikel gespeichert (oder zumindest verlinkt), so dass dieser zu einem „lebendigen Dokument" wird. ${ }^{13}$ Dies könnte man mit einem System kombinieren, welches den Autoren von Zeit zu Zeit das Einreichen neuer Versionen erlaubt - inklusive bzw. auf Basis der Kommentare.

Die Ex-post-Annotierung birgt ein gewisses subversives Potenzial in sich, denn sie lässt die Frage „Was ist Wissenschaft und wer ist Wissenschafter ${ }^{{ }^{I_{14}} \text { in }}$ einem völlig neuen Licht erscheinen. Die Frage, ob kollektive Urteile - wel-

I2 Dazu gibt es bereits Beispiele, siehe etwa <www.ecologyandsociety.org > oder $<$ www-jime.open.ac.uk>.

I3 Z. B. <www.biology-direct.com>. Siehe dazu auch: Andrew Odlyzko: Tragic Loss or Good Riddance? The Impending Demise of Traditional Scholarly Journals, in: Journal of Universal Computer Science o (I994), O, S. 7I-I22, <http:// www.jucs.org/jucs_o_o/tragic_loss_or_good>.

I4 Cary Nathenson: Electronic Publishing and the Future of Humanities Scholarship, in: Trans, Internet-Zeitschrift für Kulturwissenschaften, IO (200I), <http://www.inst.at/trans/IoNr/nathensonio.htm>. 
che sicherlich auch Beurteilungen von unqualifizierten Personen beinhalten ${ }^{15}$ - das Niveau ausgewählter Gutachter erreichen können, bleibt offen. Um zu verhindern, dass die Ex-post-Qualitätskontrolle aufgrund (noch) mangelnder Teilnahme irreführend wird, könnte man sie so gestalten, dass Kommentare erst ab einer gewissen Mindestzahl (einem Schwellenwert) angezeigt werden. Jedenfalls ist dies ein schwieriges Thema, welches eingehender Untersuchungen und Tests bedarf. ${ }^{16}$

Es gibt noch ein verwandtes Problem: Das Kommentieren könnte möglicherweise zu etwas führen, was man mit dem Begriff „Mainstreaming” umschreiben könnte. In der Hoffnung auf positive Kommentare und Bewertungen, könnten kontroverse Beiträge gänzlich vermieden werden. Auch ein solcher Effekt ist denkbar, jedoch ohne praktische Erfahrungen nur schwer abzuschätzen. Denn andererseits bekommen abweichende und innovative Meinungen durch offene Verfahren überhaupt erst die Gelegenheit zur Veröffentlichung.

(2) Bewerten (scoring): Kommentieren ist nur eine Form, auf veröffentlichte Manuskripte zu reagieren. Es hat zwei "Nachteile": Erstens müssen Kommentierende Texte verfassen, was Zeit und intensives Reflektieren erfordert. Zweitens benötigen auch die nachfolgenden Leser Zeit, um sich in den Kommentaren so zurechtzufinden, dass sie entscheiden können, ob sie einen Artikel lesen möchten. Bewerten - in Kombination mit Kommentieren, oder auch für sich genommen - wurde als Lösung für diese Probleme vorgeschlagen. Gemeint ist hier die Vergabe von Noten durch die Leser. ${ }^{17}$ Die gesammelten Bewertungen werden als Mittelwerte präsentiert (vergleichbar mit Online-Shops wie Amazon). Einige argumentieren recht radikal, dass ausge-

I5 Die Zeitschrift Philica, <www.philica.com>, etwa gibt den Nutzern je nach akademischem Status unterschiedliches Gewicht.

I6 Vgl. dazu Naboj, <www.naboj.com>, eine Open-peer-review-Plattform für Artikel des Preprint-Archivs <arxiv.org>. Ähnlich wie bei Amazon, kann man den Artikeln „Noten“ von o bis 5 geben sowie Reviews schreiben. Problematisch ist bislang allerdings die Aussagekraft dieser Bewertungen, da meist nur eine einzige vorliegt.

I7 Beispiele dafür sind Naboj und Philica (s.o.). Siehe auch: Roland Alton-Scheidl / Rupert Schmutzer / Peter Paul Sint / Gernot Tscherteu (Hrsg.): Voting, Rating, Annotation. Web4Groups and other Projects: Approaches and First Experiences, Wien / München 1997. 
reifte und demokratisch legitimierte Bewertungssysteme den „traditionellen Evaluierungseliten ${ }^{\text {"18 }}$ die Macht entziehen könnten. Für Zeitschriftenartikel wäre es denkbar, ein grobes Selektionsverfahren mit einem Kommentierungsund Bewertungsverfahren zu kombinieren, um „beleidigendes, anstößiges oder anderweitig ungeeignetes Material "ז9 auszuschließen.

Das Problem des Bewertens ist die nur schwer mögliche Teilnahmekontrolle. ${ }^{20}$ Wenn jeder, inklusive der Autoren, womöglich sogar mehr als ein Mal bewerten kann, ist das Ergebnis wahrscheinlich eher zweifelhaft, wenn nicht gänzlich unbrauchbar. Mit einem geschlossenen System aller denkbaren teilnehmenden Forschenden und mit strenger Zugangskontrolle (alle hätten einen Benutzernamen und ein Passwort), wäre ein solches semi-anonymes System technisch realisierbar. Dies würde garantieren, dass jedes Mitglied der geschlossenen Gruppe (ausgenommen der Autoren) nur eine Stimme hat, ohne dass die einzelnen Bewertungen entanonymisiert würden. Die Verwaltung der Gruppenmitgliedschaft wäre dennoch eine aufwendige Aufgabe etwa für wissenschaftliche Vereinigungen.

Ein anderes Problem ist der Umgang mit kontroversen Ergebnissen. Wenn Manuskripte ausschließlich positive oder negative Bewertungen erhalten, wäre ein System mit Durchschnittswerten sinnvoll. Was aber geschieht mit Papieren, welche die wissenschaftliche Gemeinde spalten und sowohl extrem positive als auch extrem negative Bewertungen erhalten? Wenn man nicht auch den Wert der Standardabweichung (Varianz) mit angibt, verschleiert der Durchschnittswert mehr, als er verraten würde. Eine andere Lösung für dieses Problem wäre ein differenzierteres Punktesystem, zum Beispiel als multi-dimensionales Bewertungsschema, das mehr als nur die Gesamtpunktzahl enthält. ${ }^{21}$ Die meisten Befürworter akademischer Bewertungssysteme

I8 Martin Rost: Elektronische Foren als Medien wissenschaftlicher Diskurse. Oder: Warum elektronische Foren in der vorliegenden Form nicht für den wissenschaftlichen Diskurs geeignet sind, in: Telepolis, 02.06.1998, <http://www.heise. de/tp/deutsch/inhalt/on/I389/I.html>.

I9 Varmus: PubMed Central.

20 Erste Versuche, dieses Problem in den Griff zu bekommen, wurden etwa von Philica unternommen, s.o. Fußnote 9.

2I So ermöglicht Philica scorings für die Kategorien originality, importance und overall quality. Siehe dazu auch: Stefano Mizzaro: Quality Control in Scholarly Pu- 
betrachten diese jedoch eher als Zusatz zu anderen Systemen der Qualitätskontrolle. Das gilt auch für die nächste Innovation.

(3) Zugriffs- und Zitatzählung, Analyse von Nutzungsverhalten: Die Qualität einer Publikation kann und wird auch bereits durch ihre Nutzung gemessen. In der papierbasierten Welt kann die Nutzung lediglich durch Zählung verkaufter Ausgaben errechnet werden. Diese Methode ist jedoch sehr unpräzise, da Zeitschriften und herausgegebene Bibliotheken zahlreiche Artikel enthalten und viele davon an Büchereien mit unbekannter Nutzeranzahl gehen. Darüber hinaus ist das bloße Vorhandensein einer Ausgabe ein sehr ungenauer Indikator für die tatsächliche Nutzung des Artikels. Heutzutage ist das Zählen von Zitationen eines bestimmten Artikels de facto eine gängige Praxis, mit der sich ein ganzer Wissenschaftszweig, die Szientometrie, beschäftigt. Während dies bereits einen besseren Indikator für die tatsächliche Nutzung einer Publikation darstellt, ist auch diese Methode nicht vollkommen, da die Motive für eine Zitierung unterschiedlich sein können. ${ }^{22}$

Diese innovativen Werkzeuge für Szientometrie beinhalten sowohl alte Probleme (im Falle der Zitationszählungen) als auch neue (im Falle der Zugriffszählungen und der Analyse von Nutzungsverhalten). So bleibt etwa fraglich, ob das Zählen von Zitationen oder Seitenaufrufen überhaupt etwas über die tatsächliche Qualität oder Leistung der Wissenschaft aussagt. Insbesondere was die Zugriffszählungen betrifft, gibt es immer noch eine Reihe technischer Probleme zu lösen, bevor diese überhaupt irgendeinen Nutzen haben.

blishing: A New Proposal, in: Journal of the American Society for Information Science and Technology, 54 (2003), II, S. 989-1005, <http://users.dimi.uniud. $\mathrm{it} / \sim$ stefano.mizzaro/research/papers/EJ-JASIST.pdf>.

22 Zur gängigen Kritik an der Szientometrie siehe z.B. Gerhard Fröhlich: Das Messen des leicht Meßbaren. Output-Indikatoren, Impact-Maße: Artefakte der Szientometrie?, in: Jörg Becker / Wolf Göhring (Hrsg.): Kommunikation statt Markt. Zu einer alternativen Theorie der Informationsgesellschaft, in: GMD Report 6I, Sankt Augustin 1999 (Gesellschaft für Mathematik und Datenverarbeitung), S. 27-38, <http://publica.fraunhofer.de/eprints/urn:nbn:de:ooIIb-73243I.pdf $>$ und Paul Wouters: The Citation Culture, Ph.D. thesis, Faculteit der Scheikunde, University of Amsterdam 1999, <http://web.archive.org/web/ 20050I05033804/http://www.niwi.knaw.nl/en/nerdi2/group_members/paul/ $\mathrm{publ} /$ citation/toonplaatje>. 
Es ist relativ leicht, Nutzer zum Klicken auf Internetseiten zu bewegen oder dies gar selbst manuell zu tun. Sollte die Praxis des Messens von Seitenaufrufen Bedeutung erlangen, wird es dennoch Mittel und Wege für eine adäquate Analyse der Muster von Seitenaufrufen geben. Man wird in der Lage sein, Aufrufe, die ein reales Interesse repräsentieren, zu identifizieren (etwa über die Verweildauer, d.h. die Zeit bis zum nächsten Klick) und nützliche Informationen aus den beobachteten Mustern zu extrahieren. Auch das Unterscheiden zwischen einem kurzem Anklicken eines Textes oder der genaueren Betrachtung oder gar dem Herunterladen wird mit gewissen Einschränkungen technisch möglich sein. Dennoch gibt es Hinweise darauf, dass man aus dem sog. „web download impact” relativ genau den späteren „citation impact" vorhersagen kann.

Während das Zählen anonymer Seitenaufrufe an sich noch keine Gefahr für die Privatsphäre darstellt, bringt die Analyse des Nutzungsverhalten (use tracking) ein solches Potenzial durchaus mit sich. Die für diese Zwecke verwendeten Computer müssten notwendigerweise Nutzung und Identität abgleichen können. Allerdings müsste das Offenlegen dieser sensiblen Daten ausgeschlossen werden. Stattdessen sollten nur die anonymisierten und kumulierten Daten präsentiert werden dürfen. Doch auch in diesem Fall kann eine Identifizierung der Nutzer nicht vollkommen ausgeschlossen werden, da mitunter nur wenige Personen infrage kommen. Dieses Problem besteht zwar auch bei herkömmlichen Begutachtungen, wird aber durch das Anwenden neuer Techniken (insbesondere Data-Mining) verschärft.

Abschließend lässt sich sagen, dass Szientometrie bei der Evaluierung von Papieren individuelle Expertenurteile zumindest prinzipiell ersetzen könnte. Es wurde argumentiert, dass Zitationsrankings und Expertenurteile über Wissenschafter in hohem Maße korrelieren. Sollte dieses Szenario Realität werden, würde sich der Slogan „publish or perish“ („publiziere oder gehe unter") in "get hit or get out" (,werde angeklickt oder gehe unter") weiterentwickeln. Während das Zählen von Artikeln sicherlich kein perfekter Indikator ist, erlaubt das Internet somit in Zukunft in gewisser Weise das Messen von Leserschaft. Langfristig haben Zitationsrankings und Analysen des Nutzerverhaltens das Potenzial zur vollkommenen Ersetzung der Ex-ante-Qualitätskontrolle. Mittelfristig könnten sie ein nützlicher Zusatz zu bestehenden Mechanismen sein. 


\section{QUALITÄTSKENNZEICHNUNG UND SELEKTIVE DATENBANKEN}

Leser akademischer Publikationen suchen nach Hilfsmitteln, die sie beim Filtern der kompletten Veröffentlichungen in ihrem Interessengebiet entlasten. Wie wir gesehen haben, ist die Qualitätskontrolle eines dieser Instrumente. In der papierbasierten Welt ist es der Name der Zeitschrift oder des Verlags, der zumindest den Eingeweihten eine Ahnung über die zu erwartende Qualität vermittelt: Kennt man die Zeitschrift, weiß man, welches Bewertungssystem verwendet wird. In einer bibliographischen Datenbank gibt es darüber hinaus jedoch derzeit keine weiteren Möglichkeiten, um entsprechende Informationen zu erhalten. Viele sind der Meinung, dass dieses traditionelle System dem Bedarf nach Filtern nicht optimal gerecht wird. Einige behaupten, die Filter seien mitunter zu rigide (d.h. sie filtern zu viel) und elitär. Aus dieser Sicht ist die gegenwärtige Technologie größtenteils primitiv und unzureichend, weshalb etwas Neues erforderlich ist, um Veröffentlichungen auf intelligente Weise selektieren zu können. Für die Entwicklung eines solchen Selektionsmechanismus ist die vollständige Digitalisierung eine wichtige Voraussetzung. In einer Online-Umgebung mit umfangreichen Archiven vollständiger Texte ${ }^{23}$ könnte man tatsächlich leicht über den heutigen Stand der Dinge hinausgehen. Die spezifische Kennzeichnung jedes Elementes einer Datenbank hat zwei unmittelbare Vorteile: Erstens ist für jedes Element sofort ersichtlich, welche Kontrollstufen es bereits durchlaufen hat, und zweitens kann diese Information für Suchmaschinen nutzbar gemacht werden. Folgende Elemente wurden in der Literatur für ein Kennzeichnungssystem vorgeschlagen:

(I) Bezogen auf das Qualitätskontrollsystem: Dies beinhaltet die einfache Unterscheidung bewertet vs. unbewertet; Typus und Strenge des angewandten Qualitätskontrollsystems; mit oder ohne Evaluierung ausgewählter Gutachter; abgelehnt vs. akzeptiert; Zugriffszahlen zur Messung der relativen „Signifikanz" sowie Leserbewertungen.

(2) Bezogen auf den Inhalt: Dies könnte eine Kennzeichnung für die von anerkannten Redakteuren abgeschätzte allgemeine Bedeutung beinhalten

23 Google Scholar, <scholar.google.com>, könnte sich langfristig zu einer solchen Umgebung entwickeln. 
(z.B. „Basistext" - die Information könnte dabei „dynamisch“ sein, d.h., Beschreibungen könnten später hinzugefügt oder geändert werden); Artikel-Typus hinsZusammengefasst ermöglicht eine digitale und vernetzte Publikationsumgebung die Bildung von Archiven und Datenbanken, welche nicht nur die bibliografischen Informationen und den vollständigen Text des veröffentlichten Manuskripts beinhalten, sondern auch Indikatoren über die erreichte Ebene der Qualitätskontrolle.

In einem ersten Schritt könnte man zwischen begutachteten und unbegutachteten Publikationen unterscheiden. Zudem ließe sich exakt angeben, in welchem Umfang eine Qualitätskontrolle vorgenommen wurde. Diese zusätzlichen Metadaten könnte man in Suchmaschinen integrieren, indem man Filtermechanismen verwendet, die sowohl alle als auch lediglich ausgewählte Einträge eines bestimmten Niveaus (oder Einträge einer bestimmten Kategorie) anzeigen. ${ }^{24}$

BESCHLEUNIGUNG - GLAUBWÜRDIGKEIT - PLAGIATE

Abgesehen von den neuen Qualitätskontrollverfahren beeinflusst die CyberWissenschaft indirekt auch das gesamte Qualitätskontrollsystem.

(I) Informations- und Kommunikationstechnologie hat den Begutachtungsprozess beschleunigt. Während die Gutachter sich immer noch ihre übliche Zeit nehmen, reduziert sich die Gesamtzeit der Qualitätskontrolle durch das elektronische Bereitstellen von Manuskripten zur Begutachtung und der elektronischen Weiterverarbeitung der Gutachten und der zugehörigen Korrespondenz. In einem OECD-Bericht ${ }^{25}$ wird geschätzt, dass sich die Umlaufzeit von früher einigen Monaten auf wenige Tage reduzieren lässt. Mittlerweile gibt es verbreitet Software, die zur vollstän-

24 Dies wird bei manchen offenen Zeitschriften wie „Philica“ schon getan: <www. philica.com/advancedsearch.php>.

25 OECD: Science, Technology and Industry Outlook 1998 - Chapter 7: The Global Research Village: How Information and Communication Technologies Affect the Science System, Paris: Organisation for Economic Co-operation and Development, 1998, S. 24I. 
digen Digitalisierung und Beschleunigung redaktioneller Arbeit beiträgt. Eine Rückkehr zum herkömmlichen langsamen Briefsystem erscheint indes beinahe undenkbar bzw. sogar unerwünsch ${ }^{26}-\mathrm{zu}$ sehr haben wir uns schon an die neue Geschwindigkeit gewöhnt.

(2) Aufgrund der Zunahme unbegutachteter Publikationen und des Gesamtanstiegs im Internet abrufbarer Inhalte ist die Qualität hinsichtlich ihres Status und Herkunft intransparent. Layout und Erscheinung kann im WWW irreführend sein, da es einfach ist, etwas zu kopieren und professionell wirken zu lassen. Auch der Blick auf die URL hilft hier nicht immer weiter, da man Domänennamen relativ einfach „kaufen“ kann. Somit sind Herkunft und Qualität nicht unmittelbar ersichtlich. Aber woran erkennt man dann eine glaubwürdige Quelle? Während zur Produktion wissenschaftlicher Publikationen einst eine aufwendige und teure Maschinerie (in Form eines Verlagshauses) erforderlich war, ist dies im digitalen Zeitalter nicht mehr nötig. Die Wissenschaft wird dem mit Qualitätskennzeichnung (s. o.) und mit Sammlungen glaubwürdiger Quellen begegnen müssen. So wird es den akademischen Lesern ermöglicht, Forschung in einer zuverlässigen Umgebung betreiben zu können.

(3) Die Möglichkeit des „copy and paste“, also des Kopierens und Einfügens bei digitalen Veröffentlichungen ist zwar für längeres Zitieren äußerst angenehm, erhöht aber auch die Gefahr von Plagiaten. Dies scheint insbesondere unter Studierenden, die ihre Haus- und Abschlussarbeiten mithilfe offizieller Webseiten, wissenschaftlichen Online-Artikeln und ganzen Internet-Aufsatzsammlungen verfassen, ein wachsendes Problem zu sein. Allerdings könnte diese Technik auch zu einer intensiveren und dynamischeren Kooperation zwischen den einzelnen Wissenschaftern führen und so als Fortschritt gewertet werden. Eine solche Argumentation basiert natürlich auf einem anderen Bild von Wissenschaft als dem bisherigen. Man würde kollektiv einzig nach der Weiterentwicklung des Wissens streben, unabhängig von der Person, die dazu einen Beitrag leistet. Dass diese Vorstellung mehr und mehr zur Realität wird, zeigen u.

26 Vgl. „Please do not send articles to the BMJ by post“, < <esources.bmj.com/bmj/ authors/article-submission>. 
a. die stetig wachsenden, offen zugänglichen Zeitschriften mit Creativecommons-Lizenzen. ${ }^{27}$

Es ist zu beachten, dass man dem Plagiatsproblem mit effektiven technischen Lösungen begegnen kann. Die Anwendung von Software, mit der man schnell herausfinden kann, ob große Teile eines Texts einfach aus anderen Internetquellen kopiert wurden, gehört an vielen Hochschulen längst zum Standard. Letztlich sind Plagiate aber kein technisches, sondern vor allem ein kulturelles Problem.

(4) Die elektronische Publikationsumgebung erleichtert auch Entscheidungen über Streitigkeiten zu Erstveröffentlichungen von wissenschaftlichen Erkenntnissen, da sich damit der exakte Zeitpunkt einer Veröffentlichung zumindest prinzipiell erfassen lässt. Da man diesen auch prinzipiell fälschen kann, würde dies jedoch wiederum nur im Umfeld vertrauenswürdiger Institutionen funktionieren.

\section{AUF DEM WEG ZU EINEM ZERTIFIZIERUNGSSYSTEM FÜR DIE CYBER-WISSENSCHAFT}

Dass die heutigen Qualitätskriterien akademischer Arbeit auch für das zukünftige elektronisch basierte Publikationssystem bedeutend sind, ist offensichtlich. Die oben beschriebenen Entwicklungen könnte man als umfangreichen Werkzeugkasten ansehen, mit dem man das zukünftige System errichten kann. Abhängig von den bestehenden traditionellen Qualitätskontrollen und Arbeitspraktiken, wird es zwischen den verschiedenen Disziplinen und Feldern sicherlich erhebliche Unterschiede geben. Das oft zitierte Modell der Physik ${ }^{28}$ ist gewiss kein generelles Vorbild für alle Bereiche. Dennoch sind die Unterschiede hier nicht so gravierend, dass eine Untersuchung dieses Ansatzes für die Entwicklung eines allgemeinen, nachhaltigen Zertifikationssystems keinen Sinn machen würde.

27 Vgl. <creativecommons.org>.

28 Arbeitsarchive mit Einreichung zu Zeitschriften über die Bekanntgabe der URL im Archiv. 
Führt elektronisches Publizieren zu geringerer Qualität?

Eine Argumentationsweise, die diese Frage mit "Ja“ beantwortet, ist weit verbreitet und man findet sie in verschiedenen Formen: Das Internet würde es mehr Personen ermöglichen, ihre Texte selbst online zu stellen. Dies führe zu einer Verbreitung von Vorveröffentlichungen, insbesondere derer, die noch keinerlei Kontrolle durch Außenstehende erfahren haben. Das schnellere Publizieren könne zu verfrühten Veröffentlichungen unfertiger Arbeiten verleiten. Zudem verhelfe der Computer mit der „Copy and paste“-Funktion zwar zu mehr Publikationen, nicht aber gleichzeitig auch zu mehr Gehalt. Zusammengenommen folge daraus zwangsweise eine Massenproduktion minderer Qualität.

Tatsächlich könnte es zu einer Art „Sogwirkung“ kommen, da es einfach ist, elektronisch zu publizieren und die traditionellen Qualitätskontrollen zu umgehen. Dieses Argument ist allerdings bei genauerer Betrachtung wenig überzeugend und kann durch eine Reihe von Gegenargumenten widerlegt werden:

Erstens ist „nicht begutachtet" nicht gleichzusetzen mit „keine Qualität", da die Aussicht auf spätere Veröffentlichungen Vorwirkungen mit sich bringt: Man könnte von einem „Selbstkontrolleffekt “ ${ }^{{ }_{29} 9}$ sprechen, da die „unsichtbare Hand der Begutachtung durch die Kollegenschaft" ${ }^{\prime \prime}$ auch in vermeintlich unbegutachteten Archiven präsent ist. ${ }^{31}$ Schließlich sind die Papiere für Zeitschriften bestimmt und sind daher in einer Qualität verfasst, die zukünftige Verleger und Begutachter möglichst überzeugen soll. Im Fall der Physikarchive etwa wird allgemein anerkannt, dass die Begutachtung durch die Kollegenschaft informell verläuft. Doch auch in anderen Disziplinen scheint es Sicherheitsvorkehrungen gegen minderwertige Forschung zu geben.

29 Stevan Harnad: The Invisible Hand of Peer Review, in: Nature [online], I998, <http://www.cogsci.soton.ac.uk/ harnad/nature2.html>.

30 Stevan Harnad: The Post-Gutenberg Galaxy: How to get there from here, in: Times Higher Education Supplement (THES), I995, <http://cogsci.soton. ac.uk/ harnad/THES/thes.html>.

3I Das Konzept von NowPublic, <www.nowpublic.com>, setzt dies bereits um, auch wenn es sich um keine wissenschaftliche Publikationsplattform handelt: Da User mit besseren Bewertungen mehr „Kompetenzen“ bekommen, sind sie daran interessiert, möglichst hochwertige Inhalte zu veröffentlichen. 
Zweitens erfolgt ein großer Teil der Zunahme wissenschaftlicher Publikationen nicht aufgrund des elektronischen Publizierens. Viel mehr handelt es sich um ein allgemeines Phänomen mit vielen Ursachen, insbesondere in Bezug auf den enormen weltweiten Zuwachs von Forschenden. Die Wahrnehmung, das digitale Medium könne etwas damit zu tun haben, entsteht vor allem dadurch, dass dieses den Zugang zu der Masse von Publikationen verbessert hat, insbesondere den zu den zahlreichen Arbeitspapieren. Indessen ist der verbesserte Zugang auch gleichzeitig ein wirkungsvolles Mittel gegen eine übermäßige Informationsflut. Zudem sollten wir nicht vergessen, dass die Adressaten von Arbeits- und Konferenzpapieren auch vor der Ära der Cyber-Wissenschaft sehr wohl wussten, dass es sich bei diesen um Entwürfe ohne jegliche institutionelle Garantie handelt und dass diese eben auch als solche zu behandeln sind.

Drittens sind Mehrfachveröffentlichungen nichts per se Negatives. So könnte man die Cyber-Publizierung auf innovative Weise nutzen, etwa indem man für verschiedene Zielgruppen schreibt. Es gibt aber ein noch wichtigeres Argument für unbegutachtete „Zonen“. Manche sehen die Gefahr nämlich keineswegs in der gesteigerten Textproduktion, sondern viel mehr im Gegenteil: Die sogenannte „Publikationsflut" ist ein geringeres Problem als das Ignorieren des Potenzials und der Bedeutung neuer Ideen - der eigentlichen Essenz von Wissenschaft. Wahrscheinlich geht bei der konventionellen Begutachtung ein Großteil der Informationen einfach verloren, da am Ende „eine einzige einmalige Alles-oder-nichts-Binärentscheidung" steht. ${ }^{32}$

Schließlich ist die Qualität in erster Linie eine Frage der verwendeten Qualitätskontrollsysteme und keine des Mediums, der Quantität oder der Geschwindigkeit. Daher kommt es primär auf die Publikationsnormen der jeweiligen Forschungsfelder an. Sobald ein Artikel erfolgreich eine Prüfung durchlaufen hat, ändert weder die Veröffentlichungsgeschwindigkeit etwas an seiner bestätigten Qualität noch die Tatsache, dass er zu einer immer weiter wachsenden Masse von Texten gehört.

32 Paul Ginsparg: Winners and Losers in the Global Research Village, UNESCO conference, session Scientist"s View of Electronic Publishing and Issues Raised, Paris 19.-23. Febrary 1996, <http://xxx.lanl.gov/blurb/pg96unesco.html>. 
Prinzipiell ist der Aufbau von Qualitätskontrollsystemen in der elektronischen Welt kein Problem. Die Frage ist: Werden sie etabliert? Und die vorläufige Antwort ist einfach: Warum nicht? Es gibt reichlich Belege für die Seriosität der Qualitätskontrollen elektronischer Zeitschriften (ganz zu schweigen von den elektronischen Versionen gedruckter Zeitschriften, die das traditionelle System ohnehin übernommen haben). Es gibt keinen guten Grund anzunehmen, dass man eine Qualitätskontrolle nicht auch im virtuellen Raum erfolgreich einführen könnte. Angesichts der zahlreichen neuen Möglichkeiten ist es wahrscheinlich, dass sich die traditionellen Systeme anpassen und weiterentwickeln werden. Wie wir argumentieren wollen, wird die digitale Umgebung sogar dazu beitragen, einige Unzulänglichkeiten des gegenwärtigen Systems aufzuzeigen. Insbesondere die Bewältigung des stetig wachsenden akademischen Outputs kann virtuell wesentlich effizienter erfolgen als durch den Flaschenhals des traditionellen Begutachtungssystems.

\section{Wiederherstellung und Reformierung des Peer review}

Während einige Stimmen akademische Qualitätskontrolle für gänzlich obsolet halten, scheint es einen sehr weit gehenden Konsens darüber zu geben, dass das derzeitige System eher reformiert als aufgelöst werden sollte. Die meisten Kommentatoren sehen keinen Grund, warum das Begutachtungssystem mit redaktionellen Gremien nicht in der digitalen Welt erhalten bleiben sollte. Tatsächlich gibt es bereits in allen Disziplinen Online-Publikationen mit strenger Begutachtungspolitik (z. B. die Zeitschriften von BioMedCentral).

Um die Qualität in der Cyber-Wissenschaft zu gewährleisten, werden vielfach die etablierten Praktiken aus der traditionellen papierbasierten Welt übernommen und sogar auf neue Formen akademischen Publizierens ausgeweitet. Anstatt einer simplen Reproduktion des papierbasierten Systems fordern viele eine Verbesserung und Reformierung. Das traditionelle Begutachtungssystem wird auf seinem Weg ins Zeitalter der Cyber-Wissenschaft wohl eine Modifikation und Reformation erfahren.

Einige der neuen Modelle und Möglichkeiten sind zumindest für bestimmte Akteursgruppen außerordentlich attraktiv. Die Vorteile einer vernetzten Publikationsumgebung sind mitunter derart überzeugend, dass sie von annähernd jedermann genutzt werden dürften. Insbesondere die Hilfs- 
mittel zur Beschleunigung des Qualitätskontrollprozesses werden mutmaßlich allgemein angewendet werden. Das bedeutet, dass das Verfahren der Qualitätskontrolle früher oder später komplett online verwaltet werden wird.

\section{Umsetzungsprobleme}

Auf dem Weg zur Umsetzung wird es zu einer Reihe von Problemen kommen. Hierzu ein paar Hinweise:

Qualitätskennzeichnung: Möglicherweise müssten Qualitätskennzeichnungen nicht unbedingt verpflichtend sein, da sie auch mit anderen Mitteln durchgesetzt werden könnten. Insbesondere sozialer Druck spielt dabei eine Rolle. So könnte das Fehlen von Kennzeichnungen als Zeichen mangelhafter Qualitätskontrolle interpretiert werden. Verlage dürften zur Bewerbung ihrer Produkte zur Einführung eines solchen Systems der Qualitätskennzeichnung geneigt sein.

Handhabung von überarbeiteten Versionen: Das neue Publikationssystem wird vermutlich neue Versionen von Manuskripten erlauben. Hierzu müssen die Konsequenzen für die Qualitätskontrolle diskutiert werden. Wie gravierend müssen die Änderungen in den neuen Versionen sein, um eine Neueinreichung zur Qualitätskontrolle nötig zu machen?

Belohnungssystem für Gutachter: Im Zusammenhang mit Cyber-Wissenschaft haben drei Entwicklungen zu einem erhöhten Bedarf weiterer und schnellerer Begutachtungen geführt. Erstens ist die Gesamtzahl der Publikationen gestiegen (wenn auch nur teilweise aufgrund elektronischen $\mathrm{Pu}$ blizierens). Zweitens wird in zunehmendem Maße die Auffassung vertreten, das Veröffentlichungssystem solle schnell und ohne längere Verzögerungen arbeiten (schließlich ist dies nunmehr möglich). Zuletzt muss insbesondere in einem offenen Umfeld der open peer review sichergestellt werden, dass zumindest einige der selbsternannten Gutachter vertiefend kommentieren. Manche sprechen bereits, wenn auch nur indirekt im Zusammenhang mit elektronischem Publizieren, von einer Rezensionskrise.

Ob man die Gutachter dazu verleiten könnte, mit gleich bleibender Qualität schneller zu arbeiten, ist fraglich. Einige Ansätze dazu gibt es schon, etwa das In-Aussicht-Stellen finanzieller Belohnungen. Zudem wurden jedoch 
auch weniger kostspielige Vorschläge gemacht. ${ }^{33}$ Teilweise werden als Teil des Belohnungssystems neben den publizierten Artikeln auch die Gutachten veröffentlicht. Auf diese Weise erhielte man etwas „Handfesteres“ für die wissenschaftliche Laufbahn (zugänglich für Bewerbungskommissionen etc.). Zusätzlich oder alternativ wird teilweise regelmäßig die Liste der Gutachter auf den Internetseiten von Zeitschriften und Verlagen bekannt gegeben. Während die Option finanzieller Belohnung von Cyber-Wissenschaft offensichtlich unabhängig ist, ist der zweite Vorschlag - wenn auch theoretisch in einer Print-Umgebung denkbar - wesentlich einfacher im Zeitalter elektronischen Publizierens umsetzbar.

Strategien wissenschaftlicher Vereinigungen, Bibliotheken und Universitäten: Wissenschaftliche Vereinigungen spielen bei der Implementierung neuer und der Reformierung traditioneller Systeme eine wichtige Rolle. Ein Netzwerk redaktioneller Gremien und wissenschaftlicher Vereinigungen könnte beispielsweise mit der Entwicklung der Verfahren und Kriterien betraut werden. Einige Institutionen beschäftigten sich bereits mit dem Thema, teilweise wird die Rolle der Bibliotheken oder, noch allgemeiner, der akademischen Informationsdienste betont, um die Umsetzung nationaler - oder bevorzugt internationaler - Begutachtungsstrukturen sicherzustellen. Die Involvierung wissenschaftlicher Vereinigungen und Universitäten könnte somit einen Beitrag zur Akzeptanz elektronischer Veröffentlichungen leisten.

In diesem Text haben wir sowohl das Potenzial als auch eine Reihe von Hürden (etwa die erforderliche Anpassung wichtiger Traditionen) auf dem Weg zur Erneuerung des Qualitätskontrollsystems aufgezeigt. Akademische Qualität ist prinzipiell medienunabhängig. Welches Medium auch immer - die Qualität differiert je nachdem, welches Kontrollsystem genutzt wird. Durch Cyber-Wissenschaft entstehen neue Formen der Qualitätskontrolle, die bei

33 Gutachter bekommen z. B. bei JAMA, <jama.ama-assn.org>, für schnelle Arbeit „Credits“; bei BMJ bekommen Gutachter zur Motivation ein einjähriges Abonnement der Zeitschrift sowie ein (druckbares) „Journal Activity Certificate“. 
sorgfältiger Implementierung die Kraft zur Verbesserung traditioneller Systeme mit sich bringen (insbesondere wenn sie als Zusatz oder nur zur partiellen Ersetzung traditioneller Formen eingesetzt werden). Vor allem die neuen Formen der Ex-post-Kontrolle sind in der papierbasierten Publikationswelt nicht realisierbar. In gewisser Weise könnten diese die Szientometrie revolutionieren. Zeitschriften und Archive für Arbeitspapiere werden vermutlich sowohl ex ante als auch ex post open peer review anbieten. Dies dürfte den Prozess wissenschaftlichen Publizierens weitaus kommunikativer machen, als er in seiner bisherigen Gestalt ist.

Da interaktive (im Gegensatz zu einseitiger) Kommunikation in den meisten neuen Formaten der Qualitätskontrolle eine signifikante Rolle spielt, scheint der wichtigste Faktor Zeit zu sein, nämlich die Zeit, die für eine solche verbesserte Kommunikation benötigt wird. Ob das Publizieren weiterhin von der Forderung möglichst viel zu veröffentlichen dominiert wird oder ob die Qualität die tragende Rolle spielen wird, ist kaum absehbar - und weitestgehend unabhängig von den Entwicklungen der Cyber-Wissenschaft. Im ersten Fall, wenn also weiterhin hauptsächlich die Menge der Publikationen zählt, wird nur sehr begrenzt oder überhaupt keine Zeit für zusätzliche Kommunikation in Form von offenen Kommentaren verfügbar sein. Daher würde das traditionelle System der Qualitätskontrolle wohl ins Internet verlagert werden, aber eine qualitative Metamorphose wäre weniger wahrscheinlich. Dabei läge der Einfluss der Cyber-Wissenschaft hauptsächlich darin, die Entwicklung zu beschleunigen. Im zweiten Fall bliebe dagegen mehr Zeit für Kommunikation. Dabei könnten die Potenziale voll genutzt werden und die Cyber-Wissenschaft könnte diesen Wandel wissenschaftlicher Arbeit tatsächlich beschleunigen. Welches der beiden Szenarien nun verwirklicht wird, hängt hauptsächlich von den Publikationstraditionen der verschiedenen Forschungsfelder $\mathrm{ab}$ - sowie davon, wie sich diese entwickeln.

Der Fortschritt von Qualitätskontrollsystemen in den unterschiedlichen Disziplinen wird daher nicht synchron verlaufen. Es ist gut möglich, dass die wirklich revolutionären Konzepte in manchen Feldern in naher Zukunft Gestalt annehmen werden. Insbesondere ein vereinheitlichtes Publikationsarchiv, in das alles zu Veröffentlichende als unbegutachteter Vor,,druck“ geht, erscheint attraktiv (in der Physik ist es größtenteils bereits implementiert, die Mathematik und die kognitiven Wissenschaften folgen). Kombiniert mit 
Qualitätskennzeichnung und der Verlinkung von Bewertungen und Kommentaren zu den jeweiligen Artikeln einer Datenbank, könnte so ein nutzerfreundliches, innovatives Publikationssystem entstehen, welches die Vorauswahl von Qualitätsniveaus erlauben würde. Die akademische Literatur würde zudem noch stärker in den wissenschaftlichen Kommunikationsprozess eingebettet.

Unser Titel „Peer Review 2.0“ ist somit nicht ohne Bedacht gewählt: Im Gegensatz zum traditionellen System (I.O) wird auch die Wissenschaft von den Möglichkeiten profitieren, die sich unter dem Stichwort „Web 2.0“, also einem deutlich kommunikativeren, weniger top-down organisierten, in gewisser Weise „demokratischeren“ Internet, entwickeln. 